\title{
BMJ Open Efficacy and safety of instantaneous wave-free ratio in patients undergoing coronary revascularisation: protocol for a systematic review
}

\author{
Joey S.W. Kwong, ${ }^{1}$ Sheyu Li, ${ }^{2}$ Wan-Jie Gu, ${ }^{3}$ Hao Chen, ${ }^{4}$ Chao Zhang, ${ }^{5}$ \\ Xian-Tao Zeng, ${ }^{6,7}$ Cheuk-Man Yu ${ }^{8}$
}

To cite: Kwong JS.W., Li S, Gu W-J, et al. Efficacy and safety of instantaneous wave-free ratio in patients undergoing coronary revascularisation: protocol for a systematic review. BMJ Open 2017;7:e017868. doi:10.1136/ bmjopen-2017-017868

- Prepublication history for this paper is available online. To view these files please visit the journal online (http://dx.doi org/10.1136/bmjopen-2017017868).

JS.W.K and C-MY contributed equally.

Received 21 May 2017 Revised 1 August 2017 Accepted 22 August 2017

\section{CrossMark}

For numbered affiliations see end of article.

Correspondence to Dr Joey S.W. Kwong; jswkwong@hotmail.com and Prof Cheuk-Man Yu; professorcmyu@gmail.com

\section{ABSTRACT}

Introduction Effective selection of coronary lesions for revascularisation is pivotal in the management of symptoms and adverse outcomes in patients with coronary artery disease. Recently, instantaneous 'wave-free' ratio (iFR) has been proposed as a new diagnostic index for assessing the severity of coronary stenoses without the need of pharmacological vasodilation. Evidence of the effectiveness of iFR-guided revascularisation is emerging and a systematic review is warranted.

Methods and analysis This is a protocol for a systematic review of randomised controlled trials and controlled observational studies. Electronic sources including MEDLINE via Ovid, Embase, Cochrane databases and ClinicalTrials.gov will be searched for potentially eligible studies investigating the effects of iFR-guided strategy in patients undergoing coronary revascularisation. Studies will be selected against transparent eligibility criteria and data will be extracted using a prestandardised data collection form by two independent authors. Risk of bias in included studies and overall quality of evidence will be assessed using validated methodological tools. Metaanalysis will be performed using the Review Manager software. Our systematic review will be performed according to the guidance from the Cochrane Handbook for Systematic Reviews of Interventions and the Preferred Reporting Items for Systematic Reviews and MetaAnalyses (PRISMA) statement.

Ethics and dissemination Ethics approval is not required. Results of the systematic review will be disseminated as conference proceedings and peerreviewed journal publication.

Trial registration number This protocol is registered in the International Prospective Register of Systematic Reviews (PROSPER0), registration number CRD42017065460.

\section{INTRODUCTION}

Coronary revascularisation by either percutaneous coronary intervention (PCI) or coronary artery bypass surgery (CABG) plays a crucial role in the treatment of patients with coronary artery disease (CAD) to relieve anginal symptoms and managing adverse
Strengths and limitations of this study

- We will conduct an exhaustive and systematic literature search for eligible studies in order to present a comprehensive summary of the current evidence base of the application of instantaneous 'wave-free' ratio in guiding coronary revascularisation.

- In the current era of overdiagnosis and overtreatment, findings of systematic reviews play an important role in informing clinical decisions in the management of patients with coronary artery disease undergoing revascularisation.

- Main limitation of our systematic review is that it uses study-level instead of individual patient-level data.

outcomes such as myocardial infarction (MI). Decision-making in revascularisation relies on diagnostic evidence of the presence and extent of inducible ischaemia, and only ischaemia-inducing coronary lesions-also called functionally significant stenoses or haemodynamically significant stenoses-are responsible for symptoms and thus should be selected for revascularisation. ${ }^{12}$ Therefore, accurate measurement of the functional significance of a coronary artery stenosis is required to avoid subjecting patients to inappropriate revascularisation of functionally insignificant stenoses, which can be treated successfully with medical therapy. ${ }^{12}$ Coronary angiography, a traditional imaging technique to guide revascularisation, is recognised as a flawed approach with numerous limitations in reproducibility and accuracy including marked interobserver variability and discrepancies between the diagnostic lesion severity and postmortem findings. ${ }^{3}$ Consequently, the concept of myocardial fractional flow reserve (FFR) was introduced as a new physiological index of functional severity of coronary stenoses and for determining 
ischaemia-producing coronary lesions for revascularisation. ${ }^{45}$

FFR is defined as the pressure distal to a stenosis divided by the pressure before the stenosis during coronary hyperaemia and is derived from the ratio of the mean distal artery pressure to the aortic pressure during maximal hyperaemia induced by pharmacological vasodilators such as adenosine. ${ }^{56}$ The landmark Fractional Flow Reserve versus Angiography for Multivessel Evaluation (FAME) multicentre randomised study showed that, in 1005 patients with multivessel CAD, FFR-guided PCI (using drug-eluting stents (DES) and bare-metal stents) significantly reduced the rate of the composite end point of death, non-fatal MI and repeat revascularisation at 1 year $^{7}$; the favourable effects on MI as well as the combined rate of death or MI were maintained at 2 years follow-up. ${ }^{8}$ Results of the FAME trial led to the Class 1A recommendation in the European Society of Cardiology guidelines for FFR to be used in identifying haemodynamically significant coronary lesions when evidence of ischaemia is unavailable. ${ }^{9}{ }^{10}$ Using a criterion of FFR $\leq 0.80$ to indicate functionally significant stenoses, the subsequent FAME II trial further demonstrated that FFR-guided PCI (using DES) reduced the need for urgent revascularisation among 1220 patients with stable CAD at 2 years follow-up. ${ }^{11} 12$ This body of evidence prompted the inclusion of FFR measurement as an indication in the appropriate use criteria for coronary revascularisation in patients with acute coronary syndomes (ACS) as well as patients with stable ischaemic heart disease. ${ }^{13} 14$

Despite the proven clinical and economic benefits, ${ }^{15}$ FFR-guided strategy is not without limitations. First, the fundamental assumption of FFR is that there is linearity between coronary pressure and flow during maximal hyperaemia, for which a static, stable intracoronary resistance is required; however, both assumptions do not exist in real life and represent potential errors in the measurement of FFR. ${ }^{16}$ In the context of clinical practice and patient-relevant outcomes, the need of pharmacological maximal vasodilatation for FFR-guided revascularisation poses as another limitation. The most frequently used vasodilator and current gold standard to induce hyperaemia is intravenous infusion of adenosine. ${ }^{9}{ }^{10}$ However, adenosine is associated with substantial adverse effects and patient discomfort such as dyspnoea, chest pain, headache and it is contraindicated in patients with documented allergy to adenosine or severe asthma. ${ }^{17}$ Therefore, in the real-world settings, the adoption of FFR was found to be low. ${ }^{18}$ Consequently, researchers and clinicians proposed an alternative pressure-derived index that does not require the administration of vasodilators and where measurements can be obtained during naturally constant and minimal intracoronary resistance: the instantaneous 'wave-free' ratio (iFR). ${ }^{18}$ The breakthrough ADenosine Vasodilator Independent Stenosis Evaluationis study illustrated the existence of a 'wave-free' diastolic window in the cardiac cycle when coronary resistance is naturally stable and minimal (starting $112 \pm 26 \mathrm{~ms}$ after the onset of diastole), and the iFR measured during this 'wave-free' period was found to be closely correlated with the FFR, with an optimal iFR cut-off of 0.83 for an FFR of 0.80 among a study population with a broad range of coronary stenosis severities and an iFR cut-off of 0.89 in intermediate coronary stenoses. ${ }^{19}{ }^{20}$ However, conflicting results also emerged, with data indicating that iFR provided a biased estimate of FFR on average as well as uncertain estimate of FFR in certain individual cases,${ }^{21}$ and the VERification of Instantaneous Wave-Free Ratio and Fractional Flow Reserve for the Assessment of Coronary Artery Stenosis Severity in EverydaY Practice (VERIFY) study showing that iFR at either $\leq 0.80$ or $\leq 0.83$ correlated poorly with FFR of $\leq 0.80,{ }^{22}$ which collectively limit the widespread application of iFR and led to the VERIFY study authors to advise against the use of iFR for clinical decision making in CAD patients. ${ }^{22}$ However, it is worth highlighting that, in a subsequent independent core laboratory-based retrospective analysis of VERIFY study data, a better correlation with FFR without systematic bias was demonstrated when using an iFR cut-off of $\leq 0.890 .^{23}$

The ongoing debate circulating the role of iFR as a viable guidance strategy for revascularisation has been further fuelled by the lack of outcome-based randomised controlled trials (RCTs) to investigate the clinical efficacy and safety associated with the use of iFR-guided revascularisation as compared with other guidance methods. During recent months, new trial evidence has become available and we thus aim to explore the effectiveness of iFR-guided revascularisation in CAD patients.

\section{OBJECTIVES}

This is a protocol for a systematic review to evaluate the efficacy and safety of iFR guidance in CAD patients undergoing coronary revascularisation.

\section{METHODS AND ANALYSIS}

Our systematic review will be performed according to the Cochrane Handbook for Systematic Reviews of Interventions and the Preferred Reporting Items for Systematic Reviews and Meta-Analyses (PRISMA) Statement. ${ }^{24}{ }^{25}$

\section{Eligibility criteria}

Types of studies

RCTs and controlled observational studies (including cohort and case-control studies) will be included. No restrictions on language or publication status will be imposed.

\section{Types of participants}

Patients with CAD (stable or ACS) undergoing coronary revascularisation, including PCI and CABG, will be included. 


\begin{tabular}{ll} 
Table 1 & Search \\
\hline $\mathbf{1}$ & instrategy for MEDLINE (Ovid) \\
\hline 2 & (instant* adj2 wave-free ratio).tw. \\
3 & iFR.tw. \\
4 & or/1-3 \\
5 & Coronary Artery Disease/ \\
6 & Acute Coronary Syndrome/ \\
7 & cad.tw. \\
8 & ACS.tw. \\
9 & (coronary adj3 (syndrom* or disease*)).tw. \\
10 & percutan*.tw. \\
11 & or/5-10 \\
12 & 4 and 11 \\
\hline
\end{tabular}

Types of interventions and comparators

Coronary revascularisation, as guided by iFR, is defined as the resting pressure gradient across a coronary lesion during the diastolic 'wave-free' period. The cut-off value of iFR will be as defined by the included studies. Comparators will include other diagnostic techniques for coronary revascularisation, such as coronary angiography, FFR, intravascular ultrasound (IVUS) and optical coherence tomography (OCT).

\section{Types of outcome measures}

Our primary outcomes of interest include: all-cause mortality, cardiovascular mortality, myocardial infarction (fatal and non-fatal), unplanned revascularisation. Secondary outcomes are target lesion revascularisation, stent thrombosis, coronary stenosis and adverse effects (both patient reported and physician reported).

\section{Search methods}

We will search the following electronic bibliographic databases: MEDLINE via Ovid, Embase, The Cochrane Library (Cochrane Database of Systematic Reviews, Cochrane Central Register of Controlled Trials (CENTRAL)) and University of York Centre for Reviews and Dissemination Health Technology Assessment Database from inception to September 2017, using search terms that are related to the intervention (table 1) and the search strategy will be adapted for use in each database. Trial registers, for example, ClinicalTrials.gov, will be screened for ongoing and unpublished studies. In addition, we will review safety reports released by the regulatory authorities (US Food and Drug Administration (FDA) and European Medicines Agency (EMA)). Reference lists of relevant narrative reviews and included trials will also be reviewed for additional information.

\section{Data collection and synthesis}

\section{Study selection}

Two independent authors (JSWK, SL) will screen all titles and abstracts identified from the systematic literature search; potentially eligible records will be further assessed by obtaining their full-text articles and these will again be screened by the same independent authors. Discrepancies will be resolved by consensus or by consulting a third author (CMY). A PRISMA flow diagram will be used to document our study selection process. ${ }^{25}$

\section{Data extraction}

A prestandardised data extraction form will be used to extract data from the included studies. We will extract the following information: study design; type of study population (demographics and baseline characteristics); type of study intervention and comparator(s); recruitment and study completion rates; outcomes and times of measurement; information for assessment of the risk of bias. Data will be extracted by two authors independently (JSWK, $\mathrm{CZ}$ ), and any disagreement will be resolved through discussion or by consultation with a third author (CMY). In case of missing data, we will attempt to retrieve them by contacting the study investigators.

\section{Assessment of risk of bias in included studies}

For RCTs, we will use the Cochrane Collaboration's tool for assessing risk of bias, ${ }^{24}$ focusing on the following six domains: sequence generation, allocation concealment, blinding of participants and personnel, blinding of outcome assessment, incomplete outcome data and selective outcome reporting. Risk of bias in included studies will be assessed by two independent authors (HC, WJG) and disagreements will be resolved by discussion or by consulting a third author (JSWK) where necessary. For controlled case-control and cohort studies, we will use the new tool 'Risk Of Bias In Non-randomised Studies of Interventions' (ROBINS-I) to assess the risk of bias, ${ }^{26}$ focusing on the following domains: confounding and selection of participants into the study [preintervention]; classification of the interventions (at intervention); deviations from intended interventions, missing data, measurement of outcomes and selection of the reported result [postintervention].

\section{Data synthesis}

We will use the Review Manager software (V.5.3) for data analysis. Data from RCTs and observational studies will be meta-analysed separately and presented as forest plots. Risk ratios (RRs) and the corresponding 95\% confidence intervals (CIs) will be calculated for dichotomous (binary) data; for continuous outcomes, weighted mean differences (WMDs) will be used.

Statistical heterogeneity will be explored by the $\chi^{2}$ test and quantified by the $\mathrm{I}^{2}$ statistic, with $\mathrm{p}$ value of $<0.10$ for the $\chi^{2}$ test and $\mathrm{I}^{2}$ of $\geq 50 \%$ will indicate substantial statistical heterogeneity. ${ }^{27}$ In the case of substantial heterogeneity, we will employ a random-effects model for performing meta-analysis. In other cases, a fixed-effect model will be used. Publication bias will be investigated if the number of included studies exceeds 10, using funnel plots for visual symmetry as well as the Egger's test. ${ }^{24} 27$ 
We plan to perform the following subgroup analyses if data are available and sufficient: cut-off value of iFR, types of CAD patients (stable, ACS), types of revascularisation (PCI or CABG), types of control interventions (FFR, IVUS, OCT). We will also conduct sensitivity analysis by the level of risk of bias in included studies.

Summary of findings tables will be used to illustrate the results of our assessment of the quality of evidence for each outcome according to the Grading of Recommendations, Assessment, Development and Evaluation (GRADE) framework, which considers the overall risk of bias, inconsistency, indirectness, imprecision and publication bias. $^{28}$

Should quantitative synthesis be deemed inappropriate, we will present our findings of all outcomes as narrative summaries.

\section{ETHICS AND DISSEMINATION}

Ethical approval is not required for this systematic review since data involved will be anonymous and do not concern the privacy of individual patients. Results of this systematic review will be disseminated in a peer-reviewed journal and conference proceedings.

\section{DISCUSSION}

The concept of FFR has been hailed as a pivotal paradigm shift in the management of CAD since it was first introduced in the 1990s by Pijls and colleagues, ${ }^{29} 30$ and its impact in the era of PCI has been illustrated over the years in the landmark DEFER, ${ }^{31}$ FAME and FAME 2 trials. ${ }^{7811} 12$ Moreover, vasodilators other than adenosine, for example, intravenous regadenoson, a specific $\mathrm{A}_{2 \mathrm{~A}}$ adenosine receptor agonist, ${ }^{32}$ have been investigated as viable options for FFR measurement with better safety profiles. Nevertheless, iFR poses as an attractive drug-free alternative for which investigators and researchers hope would increase adoption of coronary physiology assessment in the real world using simpler, less expensive measurement methods with better patient tolerance. ${ }^{33} 34$ The latter is especially important in clinical decision-making and longterm management and prognosis of CAD. The question of whether iFR could be a feasible 'FFR-like' index for assessing stenosis severity in CAD has been explored in numerous observational studies and randomised trials and a comprehensive systematic review assessing the currently available evidence is thus warranted.

To our knowledge, this is the first systematic review and meta-analysis investigating the efficacy and safety of iFR. A systematic review of diagnostic test accuracy found that iFR was associated with modest sensitivity and specificity, using FFR as the reference standard. ${ }^{35}$ Our systematic review of randomised and observational controlled studies will provide useful insights and shed light on the ongoing debate of the application of iFR, a new non-invasive diagnostic index, in selecting patients for coronary revascularisation. Findings of our systematic review will be obtained by comprehensive literature search and quality assessment of available evidence and, in the current era of overdiagnosis and overtreatment, may aid clinicians and relevant decision-makers in managing patients with CAD effectively.

\section{Author affiliations}

${ }^{1}$ Department of Health Policy, National Center for Child Health and Development, Tokyo, Japan

${ }^{2}$ Department of Endocrinology and Metabolism, West China Hospital, Sichuan University, Chengdu, China

${ }^{3}$ Department of Anesthesiology, Nanjing Drum Tower Hospital, Medical College of Nanjing University, Nanjing, China

${ }^{4}$ The Second Clinical College, Nanjing University of Chinese Medicine, Nanjing, China

${ }^{5}$ Center for Evidence-Based Medicine and Clinical Research, Taihe Hospital, Hubei University of Medicine, Shiyan, China

${ }^{6}$ Center for Evidence-Based and Translational Medicine, Zhongnan Hospital of Wuhan University, Wuhan, China

${ }^{7}$ Department of Urology, Zhongnan Hospital of Wuhan University, Wuhan, China

${ }^{8}$ Chiu Hin Kwong Heart Centre, Hong Kong Baptist Hospital, Kowloon, Hong Kong

Contributors JSWK and CMY conceived the idea of the study. JSWK wrote the draft of the protocol and SL, HC, WJG, CZ, XTZ and CMY revised it. JSWK and SL will search and select eligible studies; JSWK and CZ will extract data; HC and WJG will assess risk of bias, and XTZ will perform data synthesis. JSWK and CMY act as guarantors of the protocol. All authors approved the publication of the protocol.

Competing interests None declared.

Provenance and peer review Not commissioned; externally peer reviewed.

Data sharing statement Requests for unpublished data will be made available uponrequest. Please contact the first and corresponding author, JSWK, for unpublished data.

Open Access This is an Open Access article distributed in accordance with the Creative Commons Attribution Non Commercial (CC BY-NC 4.0) license, which permits others to distribute, remix, adapt, build upon this work non-commercially, and license their derivative works on different terms, provided the original work is properly cited and the use is non-commercial. See: http://creativecommons.org/ licenses/by-nc/4.0/

(C) Article author(s) (or their employer(s) unless otherwise stated in the text of the article) 2017. All rights reserved. No commercial use is permitted unless otherwise expressly granted.

\section{REFERENCES}

1. Pijls NH, Sels JW. Functional measurement of coronary stenosis. J Am Coll Cardiol 2012;59:1045-57.

2. Pijls NH, Tanaka N, Fearon WF. Functional assessment of coronary stenoses: can we live without it? Eur Heart J 2013;34:1335-44.

3. Topol EJ, Nissen SE. Our preoccupation with coronary luminology. The dissociation between clinical and angiographic findings in ischemic heart disease. Circulation 1995;92:2333-42.

4. Pijls NH, van Son JA, Kirkeeide RL, et al. Experimental basis of determining maximum coronary, myocardial, and collateral blood flow by pressure measurements for assessing functional stenosis severity before and after percutaneous transluminal coronary angioplasty. Circulation 1993;87:1354-67.

5. Pijls NHJ, de Bruyne B, Peels K, et al. Measurement of Fractional Flow Reserve to Assess the Functional Severity of Coronary-Artery Stenoses. N Engl J Med Overseas Ed 1996;334:1703-8.

6. Pijls NH, Van Gelder B, Van der Voort P, et al. Fractional flow reserve. A useful index to evaluate the influence of an epicardial coronary stenosis on myocardial blood flow. Circulation 1995;92:3183-93.

7. Tonino PA, De Bruyne B, Pijls NH, et al. Fractional flow reserve versus angiography for guiding percutaneous coronary intervention. N Engl J Med 2009;360:213-24.

8. Pijls NH, Fearon WF, Tonino PA, et al. Fractional flow reserve versus angiography for guiding percutaneous coronary intervention in patients with multivessel coronary artery disease: 2-year followup of the FAME (Fractional Flow Reserve Versus Angiography for Multivessel Evaluation) study. J Am Coll Cardiol 2010;56:177-84. 
9. Montalescot G, Sechtem U, Achenbach S, et al. Task Force MembersESC Committee for Practice GuidelinesDocument Reviewers. 2013 ESC guidelines on the management of stable coronary artery disease: the Task Force on the management of stable coronary artery disease of the European Society of Cardiology. Eur Heart $J$ 2013;34:2949-3003.

10. Windecker S, Kolh P, Alfonso F, et al. 2014 ESC/EACTS Guidelines on myocardial revascularization: The Task Force on Myocardial Revascularization of the European Society of Cardiology (ESC) and the European Association for Cardio-Thoracic Surgery (EACTS) Developed with the special contribution of the European Association of Percutaneous Cardiovascular Interventions (EAPCI). Eur Heart $J$ 20142014;35:2541-619.

11. De Bruyne B, Pijls NH, Kalesan B, et al. Fractional flow reserveguided $\mathrm{PCl}$ versus medical therapy in stable coronary disease. $N$ Engl J Med 2012;367:991-1001.

12. De Bruyne B, Fearon WF, Pijls NH, et al. Fractional flow reserveguided $\mathrm{PCl}$ for stable coronary artery disease. N Engl J Med 2014;371:1208-17.

13. Patel MR, Calhoon JH, Dehmer GJ, et al. ACC/AATS/AHA/ASE/ ASNC/SCAI/SCCT/STS 2016 Appropriate Use Criteria for Coronary Revascularization in Patients With Acute Coronary Syndromes: A Report of the American College of Cardiology Appropriate Use Criteria Task Force, American Association for Thoracic Surgery, American Heart Association, American Society of Echocardiography, American Society of Nuclear Cardiology, Society for Cardiovascular Angiography and Interventions, Society of Cardiovascular Computed Tomography, and the Society of Thoracic Surgeons. J Am Coll Cardiol 2017;69:570-91.

14. Patel MR, Calhoon JH, Dehmer GJ, et al. ACC/AATS/AHA/ASE/ ASNC/SCAI/SCCT/STS 2017 Appropriate Use Criteria for Coronary Revascularization in Patients With Stable Ischemic Heart Disease: A Report of the American College of Cardiology Appropriate Use Criteria Task Force, American Association for Thoracic Surgery, American Heart Association, American Society of Echocardiography, American Society of Nuclear Cardiology, Society for Cardiovascular Angiography and Interventions, Society of Cardiovascular Computed Tomography, and Society of Thoracic Surgeons. J Am Coll Cardiol 2017;69:2212-41.

15. Fearon WF, Bornschein B, Tonino PA, et al. Economic evaluation of fractional flow reserve-guided percutaneous coronary intervention in patients with multivessel disease. Circulation 2010;122:2545-50.

16. van de Hoef TP, Meuwissen M, Escaned J, et al. Fractional flow reserve as a surrogate for inducible myocardial ischaemia. Nat Rev Cardiol 2013;10:439-52.

17. Layland J, Carrick D, Lee M, et al. Adenosine: physiology, pharmacology, and clinical applications. JACC Cardiovasc Interv 2014;7:581-91.

18. Dattilo PB, Prasad A, Honeycutt E, et al. Contemporary patterns of fractional flow reserve and intravascular ultrasound use among patients undergoing percutaneous coronary intervention in the United States: insights from the National Cardiovascular Data Registry. J Am Coll Cardiol 2012;60:2337-9.

19. Sen S, Escaned J, Malik IS, et al. Development and validation of a new adenosine-independent index of stenosis severity from coronary wave-intensity analysis: results of the ADVISE (ADenosine
Vasodilator Independent Stenosis Evaluation) study. J Am Coll Cardiol 2012;59:1392-402.

20. Petraco R, Escaned J, Sen S, et al. Classification performance of instantaneous wave-free ratio (iFR) and fractional flow reserve in a clinical population of intermediate coronary stenoses: results of the ADVISE registry. Eurolntervention 2013;9:91-101.

21. Johnson NP, Kirkeeide RL, Asrress KN, et al. Does the instantaneous wave-free ratio approximate the fractional flow reserve? J Am Coll Cardiol 2013;61:1428-35.

22. Berry C, van 't Veer M, Witt N, et al. VERIFY (VERification of Instantaneous Wave-Free Ratio and Fractional Flow Reserve for the Assessment of Coronary Artery Stenosis Severity in EverydaY Practice): a multicenter study in consecutive patients. J Am Coll Cardiol 2013;61:1421-7.

23. Jeremias A, Maehara A, Généreux $P$, et al. Multicenter core laboratory comparison of the instantaneous wave-free ratio and resting $\mathrm{Pd} / \mathrm{Pa}$ with fractional flow reserve: the RESOLVE study. J Am Coll Cardiol 2014;63:1253-61.

24. Higgins JPT, Green S, eds. Cochrane Handbook for Systematic Reviews of Interventions Version 5.1.0. 2011: The Cochrane Collaboration. available at. www.handbook.cochrane.org. (updated March 2011accessed May 2017).

25. Moher D, Liberati A, Tetzlaff $J$, et al. Preferred reporting items for systematic reviews and meta-analyses: the PRISMA statement. PLoS Med 2009;6:e1000097.

26. Sterne JA, Hernán MA, Reeves BC, et al. ROBINS-I: a tool for assessing risk of bias in non-randomised studies of interventions. BMJ 2016;355:i4919.

27. Egger M, Davey Smith G, Schneider M, et al. Bias in meta-analysis detected by a simple, graphical test. BMJ 1997;315:629-34.

28. Balshem $\mathrm{H}$, Helfand $\mathrm{M}$, Schünemann $\mathrm{HJ}$, et al. GRADE guidelines: 3. Rating the quality of evidence. J Clin Epidemiol 2011;64:401-6.

29. Pijls NH, van Son JA, Kirkeeide RL, et al. Experimental basis of determining maximum coronary, myocardial, and collateral blood flow by pressure measurements for assessing functional stenosis severity before and after percutaneous transluminal coronary angioplasty. Circulation 1993;87:1354-67.

30. Pijls NH, De Bruyne B, Peels K, et al. Measurement of fractional flow reserve to assess the functional severity of coronary-artery stenoses. N Engl J Med 1996;334:1703-8.

31. Bech GJ, De Bruyne B, Pijls NH, et al. Fractional flow reserve to determine the appropriateness of angioplasty in moderate coronary stenosis: a randomized trial. Circulation 2001;103:2928-34.

32. Nair PK, Marroquin OC, Mulukutla SR, et al. Clinical utility of regadenoson for assessing fractional flow reserve. JACC Cardiovasc Interv 2011;4:1085-92.

33. Kern MJ. An adenosine-independent index of stenosis severity from coronary wave-intensity analysis: a new paradigm in coronary physiology for the cath lab? J Am Coll Cardiol 2012;59:1403-5.

34. Sen S, Escaned J, Francis DP, et alJ Am Coll Cardiol 2012:59:1917-8.

35. Man W, Hu J, Zhao Z, et al. Diagnostic performance of instantaneous wave-free ratio for the evaluation of coronary stenosis severity confirmed by fractional flow reserve: A PRISMAcompliant meta-analysis of randomized studies. Medicine 2016;95:e4774. 